\title{
Evolução clínica a longo prazo, correlacionando a presença de bloqueios da condução intraventricular em pacientes chagásicos e não chagásicos assintomáticos
}

\author{
Long-term follow up of asymptomatic chagasic individuals with intraventricular \\ conduction disturbances, correlating with non-chagasic patients
}

João Pimenta, Ney Valente e Manoel Miranda

\begin{abstract}
Resumo Estudo prospectivo para avaliar o potencial evolutivo, a longo prazo, dos transtornos da condução intraventricular em indivíduos chagásicos e não chagásicos assintomáticos. Foram submetidos a estudo eletrofisiológico, por metodologia convencional, 84 indivíduos portadores de bloqueio de ramo direito ou esquerdo, 55 chagásicos e 29 não chagásicos. A idade média foi de 45 anos nos chagásicos e 63 nos não chagásicos $(p<0,001)$. Posteriormente, foram seguidos ambulatorialmente. $O$ estudo eletrofisiológico nâo mostrou diferenças estatisticamente significantes entre as médias das freqüências cardíacas basais dos dois grupos, bem como entre as médias dos intervalos $A H$, do tempo máximo de recuperação do nódulo sinusal e do ponto de Wenckebach. A média do intervalo HV mostrou-se maior no grupo NCH $(p<0,001)$. Após seguimento médio de 121 meses nos chagásicos e 94 nos não chagásicos (NS), observouse que a mortalidade total entre os primeiros foi de 20 (36,7\%) pacientes e no segundo 9 (31\%) (NS). Morte de causa cardíaca e morte súbita foi documentada em $17(85 \%)$ indivíduos chagásicos e $3(33,3 \%)$ nos não chagásicos $(p<0,05)$. Morte súbita ocorreu em $10(50 \%)$ nos chagásicos e não observada entre os não chagásicos $(p<0,01)$. Conclui-se que: 1 . Os indivíduos chagásicos apresentaram maior mortalidade cardíaca súbita ou não súbita, que os indivíduos não chagásicos. 2. Os parâmetros eletrofisiológicos estudados não tiveram valor prognóstico quanto à mortalidade, com a ressalva de que a estimulação ventricular não foi realizada.
\end{abstract}

Palavras-chaves: Doença de Chagas. Bloqueio intraventricular. Morte súbita.

\begin{abstract}
This prospective study was designed to evaluate intraventricular conduction disturbances in asymptomatic patients with Chagas' disease, and patients with primary sclerosis of the conducting system of the heart (Lev-Lenègre's disease). Eighty-four asymptomatic individuals with right- or left-bundle branch block were submitted to electrophysiological evaluation and longterm follow-up. Fifty-five had positive serologic blood tests for Chagas' disease and 29 had LevLenègre's disease. Mean-age of 45 years in chagasic and 63 in non-chagasic patients $(p<0.001)$. There were 54 (98.2\%) with right-bundle branch block in chagasic and 14 (48.3\%) among nonchagasic patients $(p<0.001)$. Sinus nodal and atrioventricular nodal functions presented no differences in either group. However, mean HV interval was greater in the $\mathrm{NCH}$ than in the $\mathrm{CH}$ group $(p<0.01)$. After a mean follow-up of $121 \pm 63.75$ months among chagasic and $94.97 \pm$ 67.55 months among non-chagasic patients, total mortality was 20 (36.6\%) patients in chagasic and 9 (31\%) among non-chagasic patients (NS). Cardiac death was recorded in 17 (85\%) individuals among chagasic and $3(33.3 \%)$ among non-chagasic patients $(p<0.05)$. Sudden death occurred in 10 (50\%) among chagasic and was not observed in non-chagasic patients $(p<0.01)$. In conclusion: 1. Asymptomatic chagasic individuals with intraventricular conduction disturbances showed higher cardiac mortality, sudden or non-sudden death in relation to non-chagasic individuals. 2. The evaluated electrophysiological parameters were of no prognostic value in relation to mortality, although programmed ventricular stimulation was not performed.
\end{abstract}

Key-words: Chagas' disease. Intraventricular conduction disturbances. Sudden death.

Serviço de Cardiologia, Hospital do Servidor Público Estadual, São Paulo, SP.

Endereço para correspondência: Dr. João Pimenta. R. das Camélias 357, 04048-060 São Paulo, SP.

Tel: 55 11 276-3856; Fax: 5511 5581-5618

Recebido para publicação em 3/2/98. 
Entre as formas clínicas da doença de Chagas, inclui-se a cardíaca que comumente encerra comprometimento do sistema éxcito-condutor do coração, levando a distúrbios da condução atrioventricular (AV) ou intraventricular - os bloqueios de ramo - numa fase ainda assintomática da doença. O bloqueio completo do ramo direito (BCRD) é um achado freqüente ${ }^{11} 17^{3134}$, sendo considerado alteração peculiar da cardiopatia chagásica quando associado ao desvio do ÂQRS para a esquerda ${ }^{15} 33$. O BCRD pode ser observado em 13 a $55 \%$ dos indivíduos soropositivos ${ }^{113133} 34$, prevalência esta que varia segundo a população estudada. No caso do bloqueio completo do ramo esquerdo (BCRE), esses estudos apontaram que sua prevalência é menor, situando-se entre 2 a $5 \%$. O acompanhamento desses indivíduos tem revelado o aparecimento, também de bloqueios atrioventriculares, bem como de insuficiência cardíaca, e taxas significativas de mortalidade cardíaca, a qual deve-se principalmente à morte súbita23 2939 .

O presente trabalho tem como objetivo relatar um estudo prospectivo sobre o potencial clínico e eletrofisiológico cardíaco de portadores assintomáticos de cardiopatia chagásica crônica com determinados bloqueios de ramo, em comparação com não chagásicos também portadores de transtornos da condução intraventricular. Objetiva ainda verificar o papel do estudo eletrofisiológico invasivo na discriminação desses diferentes grupos quanto ao potencial para aparecimento de bloqueio $\mathrm{AV}$, a necessidade de implante de marcapasso definitivo, mortalidade total e de causa cardíaca.

\section{MATERIAL E MÉTODOS}

Características do estudo. Prospectivo, com população recrutada de forma consecutiva. Os indivíduos chagásicos (grupo $\mathrm{CH}$ ), diagnosticados por exames sorológicos (reações de fixação de complemento, imunofluorescência e hemaglutinação), foram recrutados entre doadores de sangue do Hospital do Servidor Público Estadual, São Paulo, SP e entre candidatos a emprego em empresas da grande São Paulo, sob a condição de não apresentarem qualquer sintomatologia relacionada com possível cardiopatia. Os indivíduos não chagásicos (grupo $\mathrm{NCH}$ ), portadores de esclerose idiopática do sistema de condução ${ }^{19} 2032$, foram recrutados dentre os que procuraram o Serviço de Cardiologia do mesmo Hospital para uma avaliação clínica tipo "check-up", como pré-operatório ou por serem hipertensos, dislipêmicos ou diabéticos, todos controlados apenas com dieta.

Todos os indivíduos incluídos no estudo foram submetidos a exame clínico, eletrocardiograma de repouso, radiografia de tórax e estudo eletrofisiológico, e posteriormente seguidos ambulatorialmente. Foram estudados 84 pacientes, sendo $55(65,5 \%)$ pertencentes ao grupo $\mathrm{CH}$ e $29(34,5 \%)$ ao $\mathrm{NCH}$. A Tabela 1 mostra as características dos indivíduos do grupo $\mathrm{CH}$, enquanto que a Tabela 2, as do grupo $\mathrm{NCH}$. O estudo eletrofisiológico foi realizado por meio de metodologia previamente descrita ${ }^{27}{ }^{37}$.

Critérios de inclusão. Qualquer idade, raça, e de ambos os sexos, assintomáticos do ponto de vista cardiovascular, sem antecedentes de qualquer cardiopatia orgânica (a não ser o distúrbio intraventricular), eletrocardiograma de repouso de 12 derivações com ritmo sinusal, condução AV 1:1, intervalo $P R \leq 0,20$ s e bloqueio completo de ramo de qualquer tipo, área cardíaca normal à radiografia de tórax. Nenhum paciente estava recebendo qualquer fármaco cardioativo ou qualquer medicamento que, conhecidamente, pudesse alterar as condições eletrofisiológicas do sistema éxcito-condutor cardíaco. Houve aceitação por escrito para se submeter ao estudo eletrofisiológico invasivo.

Critérios de exclusão. Presença de bloqueios de ramo intermitentes, de causa congênita, póscirúrgica, traumática e reversível, ou de qualquer etiologia que não fosse a esclerose idiopática do sistema de condução ou doença de Chagas, pacientes na classe funcional II ou acima, da "New York Heart Association", presença de doença consumptiva, pneumopatia crônica descompensada, diabetes controlado com medicamentos, alterações hidroeletrolíticas, seqüelas de infarto do miocárdio, coronariopatia evidente, ou com qualquer condição que pudesse predizer curto período de seguimento.

Seguimento clínico. A data de entrada no protocolo foi a do estudo eletrofisiológico e a do encerramento, a da última consulta ambulatorial, do último contato telefônico ou a do óbito. O seguimento clínico foi feito em regime ambulatorial, com consultas periódicas semestrais, a menos que surgisse alguma intercorrência que necessitasse de antecipação. Em caso de falta de comunicação 
por mais de um ano, os indivíduos eram contatados por telefone. Em cada consulta ambulatorial eram realizados exame clínico geral e eletrocardiograma de 12 derivações em repouso. $\mathrm{Na}$ eventualidade de aparecimento de sintomas eram solicitados outros exames ou tomadas as medidas terapêuticas necessárias. Nenhum paciente recebeu antiarrítmicos ou outros fármacos cardioativos, exceto os que desenvolveram insuficiência cardíaca, que receberam digital e/ou diuréticos. No contato telefônico, caso tivesse ocorrido óbito, eram investigadas as condições que precederam o evento, o modo como ele ocorreu ou se houve internação em algum hospital. Nesta eventualidade, o hospital era contatado para obtenção de informações mais detalhadas. $\mathrm{O}$ grupo $\mathrm{CH}$ foi seguido durante $121,07 \pm 63,75$ meses (variando entre 2 e 222, com mediana de 118), enquanto que o grupo $\mathrm{NCH}$ foi seguido durante $94,97 \pm$ 66,93 meses (variando entre 5 e 223, com mediana de 74), diferença não significante (NS).

Tratamento estatístico. Para a análise dos resultados foram utilizados testes não paramétricos, levando-se em consideração a natureza das variáveis estudadas. Foram aplicados os seguintes testes: do qui-quadrado para tabelas $2 \times 2$, sendo que em alguns casos, aplicou-se o teste exato de Fisher. Teste de Mann-Whitney para duas amostras independentes. Em todos os testes foi fixado em 0,05 ou $5 \%(\alpha \leq 0,05)$ o nível de rejeição da hipótese de nulidade. Os dados serão apresentados em média \pm desviopadrão, quando necessário.

\section{RESULTADOS}

Nas Tabelas 1 e 2 observa-se que, no grupo $\mathrm{CH}$, a média de idade foi de 45,75 $\pm 11,20$ (variação entre 25 e 76 anos) e no grupo $\mathrm{NCH}$ foi de $63,0 \pm 12,72$ (variando entre 36 e 87 anos) $(p<0,001)$. A distribuição por faixa etária encontrase disposta na Tabela 3. Havia significantemente maior número de indivíduos abaixo de 60 anos de idade no grupo $\mathrm{CH}$ que no $\mathrm{NCH}(\mathrm{p}<0,001)$. No grupo $\mathrm{CH}$, havia $33(60 \%)$ homens e $22(40 \%)$ mulheres e, no $\mathrm{NCH}, 17$ (58,6\%) homens e 12 $(41,4 \%)$ mulheres (NS).

Doenças associadas. Megacolo, megaesôfago ou ambos foram encontrados em 14 (25,4\%) indivíduos do grupo $\mathrm{CH}$ e hipertensão arterial essencial e hiperglicemia controladas apenas com dieta foram encontradas em 6 e 3 indivíduos do grupo $\mathrm{NCH}$, respectivamente. Nos 20 restantes deste grupo, o bloqueio de ramo foi um achado eletrocardiográfico.

Morfologia dos complexos QRS. No grupo $\mathrm{CH}$ (quadro I), havia $54(98,2 \%)$ pacientes portadores de BCRD, isolado em 15, associado a hemibloqueio anterior esquerdo (HBA) em 37 e a hemibloqueio posterior esquerdo (HBP) em 2. No grupo $\mathrm{NCH}$ (quadro II), havia $15(51,7 \%)$ indivíduos com BCRD, isolado em 4 e associado a HBA em 11, e $14(48,3 \%)$ com BCRE. A análise estatística quanto à prevalência de $\mathrm{BCRD}$ em cada grupo mostrou diferença significante $(p<0,001)$.

Parâmetros eletrofisiológicos. As médias dos parâmetros eletrofisiológicos analisados em ambos os grupos não apresentaram diferenças estatisticamente significantes, exceto a média do intervalo $\mathrm{HV}$, que se mostrou maior no grupo $\mathrm{NCH}$ $(p<0,01)$. Essas médias, quando analisadas somente nos portadores de BCRD, isolado ou associado a HBA, também não mostraram diferenças significantes, exceto o tempo de recuperação do nódulo sinusal (TRNS), que se mostrou significantemente maior no grupo $\mathrm{CH}$, nos portadores de BCRD isolado $(p<0,05)$. A presença de disfunção do nódulo sinusal, do nódulo AV e simultaneamente do nódulo AV e do sistema His-Purkinje (lesões em série no sistema juncional AV) não apresentaram diferenças significantes. Porém, disfunção do sistema HisPurkinje foi significantemente maior no grupo $\mathrm{NCH}(\mathrm{p}<0,01)$.

Mortalidade. No grupo $\mathrm{CH}$ foram registrados $20(36,3 \%)$ óbitos e 9 (31\%), no grupo NCH (NS). A mortalidade de causa cardíaca foi constatada em 17 (85\%) indivíduos no grupo $\mathrm{CH}$ e em 3 $(33,3 \%)$, no grupo $\mathrm{NCH}(\mathrm{p}<0,05)$. A morte súbita ocorreu em 10 (50\%) indivíduos do grupo $\mathrm{CH}$, não sendo observada no grupo $\mathrm{NCH}(\mathrm{p}<0,01)$. A Figura 1 mostra as curvas atuariais de sobrevida dos dois grupos, não se observando diferença significante entre ambas. Não houve relação estatisticamente significante da mortalidade com relação ao sexo, à faixa etária, à presença de bloqueio do ramo direito, às alterações do nódulo sinusal, à disfunção do sistema His-Purkinje e às alterações simultâneas no nódulo AV e no sistema His-Purkinje. Contudo, a mortalidade de causa cardíaca no grupo $\mathrm{NCH}$ correlacionou-se de forma significante com o aumento do TRNS $(p<0,05)$, embora o número de indivíduos avaliados tenha sido pequeno. Com relação às alterações do intervalo $\mathrm{AH}$ houve uma 


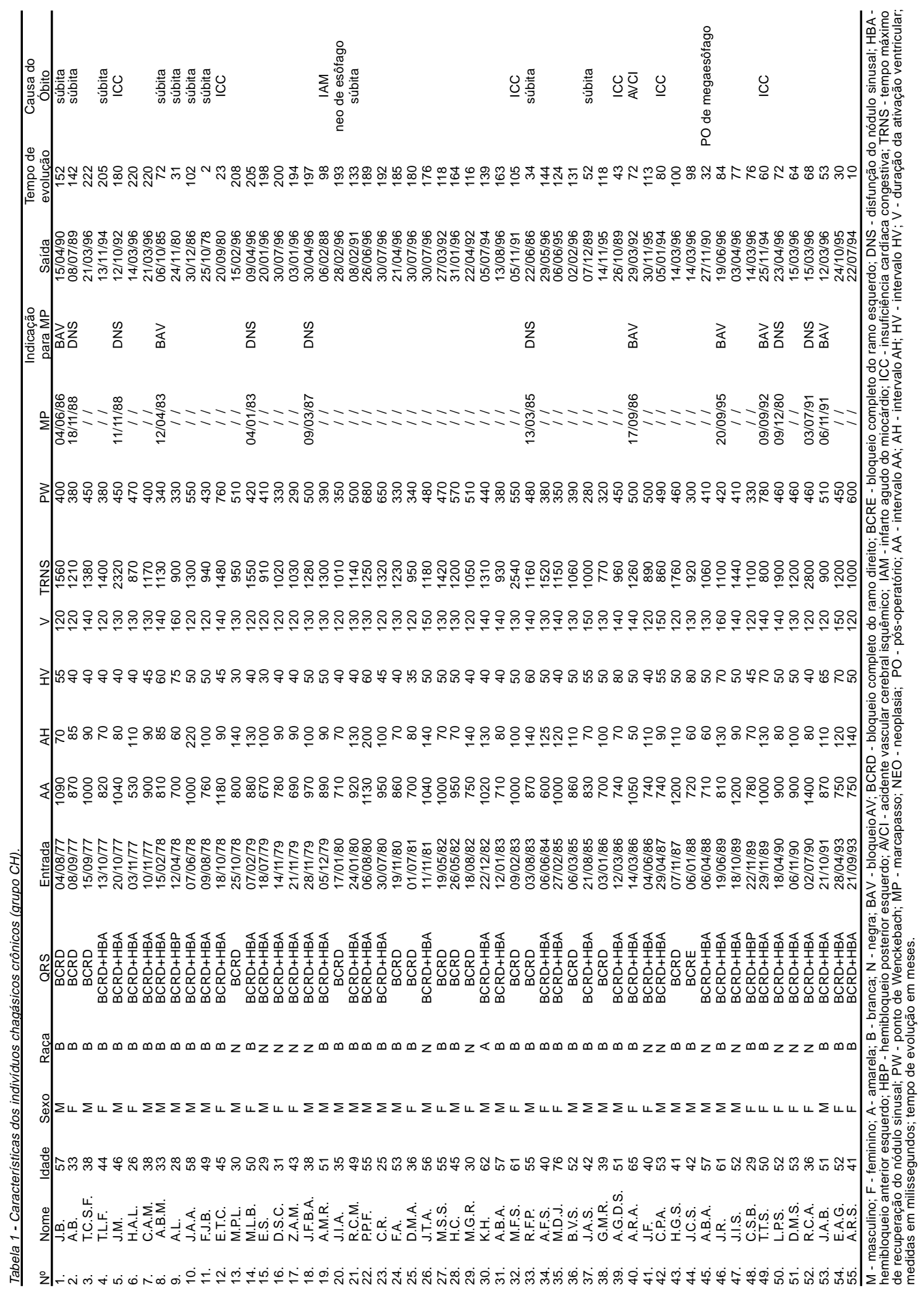




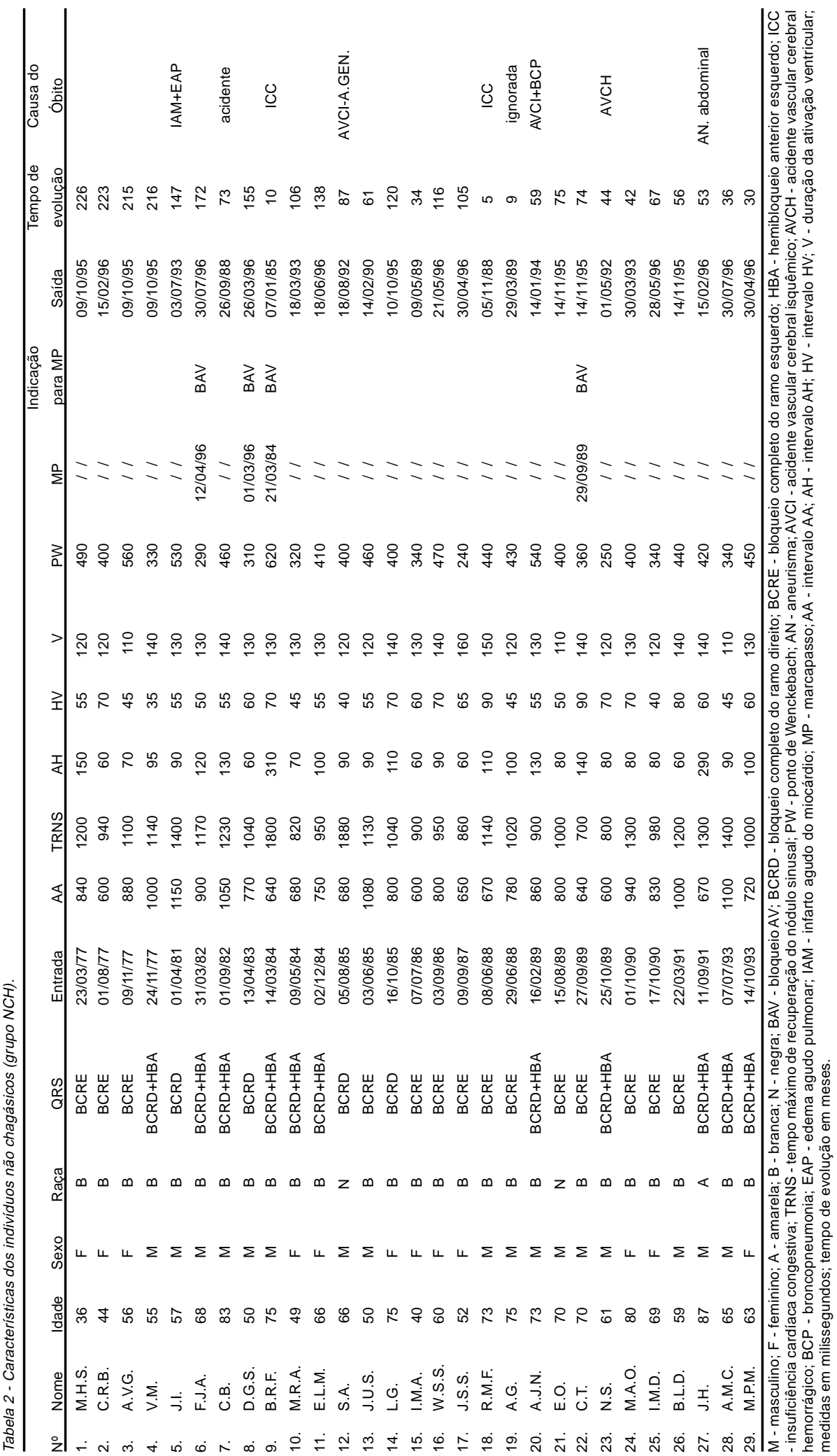


Tabela 3 - Características dos indivíduos chagásicos $(\mathrm{CH})$ e não chagásicos $(\mathrm{CH})$ por faixa etária no momento da entrada no estudo e mortalidade (morte de causa cardíaca entre parênteses).

\begin{tabular}{lrccccc}
\hline Idade $\mathrm{N}$ & \multicolumn{3}{c}{$\mathrm{CH}$} & \multicolumn{3}{c}{$\mathrm{NCH}$} \\
\cline { 2 - 7 } anos & $\mathrm{N}$ & mortalidade & $\%$ & $\mathrm{~N}$ & mortalidade & $\%$ \\
\hline$\leq 40$ & 19 & $4(3)$ & 21,0 & 2 & - & - \\
$41-50$ & 12 & $7(7)$ & 58,3 & 2 & - & - \\
$51-60$ & 19 & $7(6)$ & 36,8 & 8 & $1(1)$ & 12,5 \\
$\geq 60$ & 5 & $2(1)$ & 40,0 & 17 & $7(2)$ & 47,0 \\
\hline total & 55 & $20(17)$ & 36,4 & 29 & $9(3)$ & 31,0 \\
\hline
\end{tabular}

associação significante entre a sobrevida e a média dos intervalos $\mathrm{AH}$ maiores no grupo $\mathrm{CH}$, ocorrendo o inverso, no grupo $\mathrm{NCH}$, fato não observado quando se analisou a mortalidade de causa cardíaca. O ponto de Wenckebach não mostrou associação significante com a mortalidade total. Quando se analisou a mortalidade de causa cardíaca, esse parâmetro se relacionou de forma significante com a mortalidade apenas no grupo $\mathrm{NCH}$, embora o número de indivíduos tenha sido limitado para melhor avaliação $(p<0,05)$.

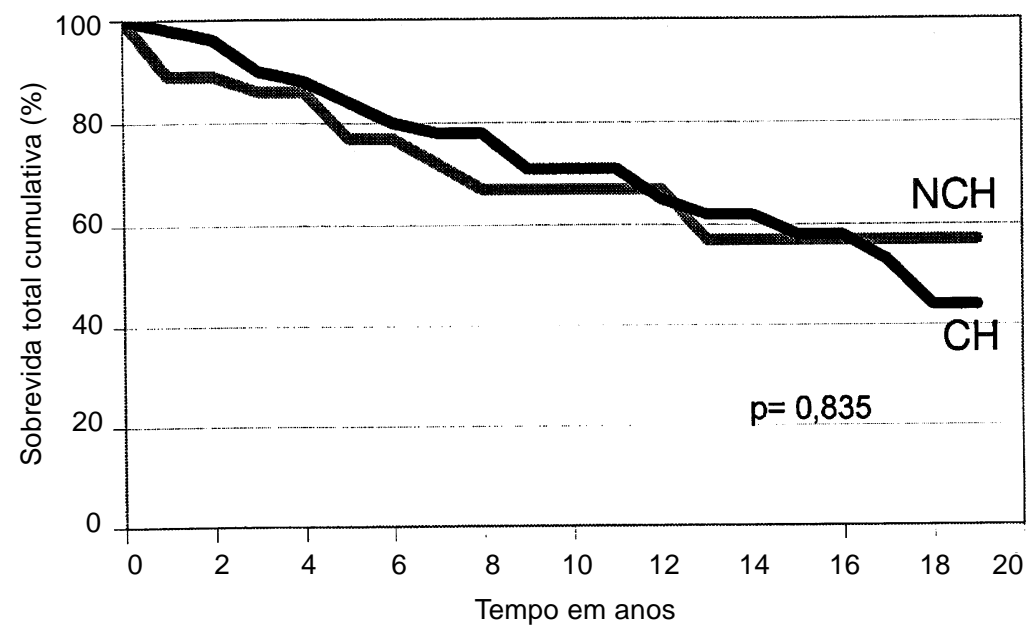

Figura 1 - Curva de sobrevida total cumulativa dos indivíduos chagásicos $(\mathrm{CH})$ e nâo chagásicos (NCH). Observar que não houve diferenca estatiticamente significante entre a mortalidade de ambos os grupos, devendo, contudo, considerar que a faixa etária é diferente.

Necessidade de implante de marcapasso. Não houve diferença estatisticamente significante entre os chagásicos e os não chagásicos quanto à necessidade de implante de marcapasso por qualquer causa. As indicações para implante de marcapasso, em ambos os grupos, bem como seus parâmetros eletrofisiológicos, estão nas Tabelas 1 e 2, podendo observar-se que, no grupo $\mathrm{CH}, 6$ tiveram indicação para implante secundário a bloqueio $\mathrm{AV}$ e 7 por disfunção do nódulo sinusal, sendo que, no grupo $\mathrm{NCH}$, os 4 foram secundários a bloqueio AV. Não houve relação estatisticamente significante da necessidade de implante de marcapasso com o sexo, faixa etária, presença de BCRD, alterações do nódulo $\mathrm{AV}$, lesões simultâneas no nódulo AV e sistema His-Purkinje e ponto de Wenckebach. Entretanto, o TRNS mostrou que os valores maiores no grupo $\mathrm{CH}$ correlacionaram-se de forma significante com a evolução para disfunção do nódulo sinusal e conseqüente necessidade de implante de marcapasso $(p<0,05)$. Esta variável não pôde ser analisada no grupo $\mathrm{NCH}$, porque neste grupo não houve necessidade de implante de marcapasso devido a esta disfunção. O mesmo ocorreu com as alterações do sistema His-Purkinje cujos valores do intervalo HV dos dois grupos que evoluíram ou não para bloqueios 
AV e que receberam implante de marcapasso, mostraram correlação estatisticamente significante apenas no grupo $\mathrm{CH}$, sugerindo que pacientes chagásicos com intervalo HV aumentado apresentam maior chance de desenvolverem bloqueio $\mathrm{AV}$. No grupo $\mathrm{NCH}$, esta relação não se mostrou significante. Nos dois grupos, o número limitado de indivíduos que evoluíram para bloqueio AV não permite uma análise mais acurada.

\section{DISCUSSÃO}

A presença de bloqueio de ramo é considerada um fator de mau prognóstico ${ }^{4,18}$. No entanto, alguns trabalhos com grande número de casos, vêm sugerir que a presença de bloqueio de ramo, quando única alteração no quadro patológico, pode não ser preditora de má evolução ${ }^{2} 73$. Tais trabalhos estudaram indivíduos assintomáticos e sintomáticos, apresentando pré-síncope, síncope ou tonturas. Porém, quando se associa a outro tipo de cardiopatia (hipertrofia miocárdica, seqüela de infarto do miocárdio ou disfunção ventricular esquerda), o prognóstico pode mudar de forma marcante. Isso se torna mais importante, quando se encontram bloqueios de ramo associados a desvios do ÂQRS9141625 29. Esses estudos revelaram que indivíduos portadores de bloqueios de ramo com corações estruturalmente normais têm boa evolução a longo prazo e que os portadores de BCRE têm uma evolução com maior morbimortalidade em relação aos com BCRD, pois, na grande maioria dos casos, associa-se a algum tipo de cardiopatia.

Alguns estudos semelhantes já existem com pacientes chagásicos crônicos. A assim chamada forma indeterminada ${ }^{12}$ parece ter pequenas alterações estruturais e boa evolução a longo prazo, embora tenha uma maior incidência de aparecimento de distúrbios da condução e insuficiência cardíaca em relação à população normal ${ }^{1323}$. Porém, quando se instala o bloqueio de ramo, geralmente BCRD isolado ou associado a hemibloqueios, a evolução é pior ${ }^{13}{ }^{23}$. Todos esses estudos foram conduzidos com avaliações clínicas, eletrocardiográficas e radiológicas, em populações heterogêneas.

Em população não chagásica, a presença de alterações no intervalo $\mathrm{AH}$ não parece representar grande importância quanto à evolução clínica ${ }^{8}$, ocorrendo evolução para bloqueios $\mathrm{AV}$ em $3 \%$ dos que previamente tinham intervalo $\mathrm{AH}$ normal e em $10 \%$ dos que tinham intervalo $\mathrm{AH}$ aumentado, embora não significando necessidade de implante de marcapasso. Porém, alterações no sistema His-Purkinje sugerem evolução diferente, já que lesões distais ao local onde se registra o potencial do feixe de His, em portadores de bloqueio bifascicular (BCRD associado a hemibloqueios), têm percentagem maior de evolução para graus avançados de bloqueio $\mathrm{AV}$, pois, $46,6 \%$ dos que tinham intervalo HV aumentado desenvolveram bloqueios AV de $2^{\circ}$ e $3^{\circ}$ graus $^{10}$. Achados semelhantes foram relatados por outros ${ }^{16}$, que mostraram menor mortalidade entre os que implantaram marcapasso.

A análise desta casuística revela que no grupo $\mathrm{NCH}$ havia maior número de portadores de alterações no sistema His-Purkinje. Estas alterações poderiam estar presentes devido à faixa etária maior neste grupo, ou porque a doença que as provocou tenha afetado de forma mais específica o tecido éxcito-condutor cardíaco. Contudo, isto não indicou pior evolução destes para bloqueios $\mathrm{AV}$ de $2^{\circ}$ ou $3^{\circ}$ graus, maior necessidade de implante de marcapasso ou maior mortalidade; o evento mortalidade total ou de causa cardíaca, não mostrou diferença significante entre os grupos. Deve-se considerar que o grupo $\mathrm{NCH}$ apresentou uma faixa etária significantemente maior, bem como maior número de indivíduos portadores de BCRE, fator preditor de maior morbi-mortalidade evolutiva ${ }^{2}$. Como mostrou evolução semelhante aos chagásicos, que tinham menor faixa etária e menor prevalência de BCRE, os achados do presente estudo indicam maior gravidade das alterações encontradas nos chagásicos crônicos com bloqueio de ramo.

A morte súbita ocorre com muita freqüência na cardiopatia chagásica crônica ${ }^{5130}$ e os seus fatores preditores estão incompletamente definidos. Na presente casuística, deve-se considerar a alta incidência de indivíduos falecidos subitamente, tendo sido observado que, dos 20 óbitos no grupo $\mathrm{CH}, 10$ (50\%) ocorreram de forma súbita, enquanto que no grupo $\mathrm{NCH}$ não se registrou tal tipo de evento. Mesmo comparando com outros estudos de não chagásicos, em que se encontrou alta incidência de morte súbita ${ }^{4}$, as características clínicas não são comparáveis com a presente casuística, não só pela faixa etária, muito mais baixa entre os 
chagásicos, como as cardiopatias associadas. Esses estudos avaliaram pacientes com bloqueios bifasciculares, mas com angina, seqüelas de infarto do miocárdio e insuficiência cardíaca ${ }^{475}$, que foram critérios de exclusão do presente estudo. Como esta investigação seguiu um número significativo de pacientes por um tempo longo, a alta incidência de morte súbita no grupo $\mathrm{CH}$ sugere que a presença de BCRD correlaciona-se com evolução para morte súbita. Deve-se aqui considerar que a morte súbita nos indivíduos chagásicos crônicos assintomáticos é interpretada como conseqüente a arritmias ventriculares $^{22}{ }^{30}$. Porém, sabe-se que ela pode ocorrer devido a episódios de assistolia, tromboembolia pulmonar ou a outras causas não cardíacas $^{26} 30$.

Neste estudo não foi observada diferença de mortalidade entre os dois grupos quanto às alterações eletrofisiológicas do nódulo sinusal, embora o número de indivíduos com essas alterações tenha sido pequeno. Em relação à mortalidade de causa cardíaca, houve correlação significante apenas nos indivíduos do grupo $\mathrm{NCH}$, em que o TRNS foi maior entre aqueles que evoluíram com óbito. Porém, o número de óbitos de causa cardíaca também foi pequeno, o que não permite conclusões definitivas. A evolução para formas sintomáticas e que vieram a necessitar de marcapasso foi maior entre os indivíduos chagásicos que já apresentavam sinais de disfunção do nódulo sinusal (não houve necessidade de implante de marcapasso por disfunção do nódulo sinusal entre os não chagásicos). Isto sugere que a documentação desta disfunção é importante na cardiopatia chagásica e que poderia identificar candidatos para futuro implante de marcapasso.

Os indivíduos dos dois grupos que tinham disfunção do nódulo AV apresentaram comportamento semelhante quanto a necessidade de marcapasso. Por outro lado, houve uma associação entre a mortalidade total com menores tempos de condução no nódulo $\mathrm{AV}$, embora não tenha havido relação com a mortalidade de causa cardíaca. Esse fato se mostra intrigante, podendo-se especular que o aumento do intervalo $\mathrm{AH}$ represente um maior comprometimento do nódulo AV. A constatação de intervalo $\mathrm{AH}$ menor entre os que faleceram pode indicar ação adrenérgica e que o sistema nervoso autônomo (simpático) representou um importante papel, provocando um aumento da mortalidade. Por outro lado, o maior intervalo $\mathrm{AH}$ entre os sobreviventes pode ser secundário a uma alteração do sistema nervoso autônomo, com maior efeito vagal sobre essa estrutura, dificultando a ação do simpático, e daí, menor mortalidade. Este fato é de difícil interpretação, pois, quando se consideram as alterações funcionais do nódulo AV, que englobam o tempo de condução intranodal AV (intervalo $A H$ ) e o ponto de Wenckebach, não se encontrou associação significante entre essas alterações e a mortalidade, principalmente com a de causa cardíaca. Embora se tenha conhecimento de que a maioria dos pacientes chagásicos crônicos apresentam alterações mais freqüentes no nódulo AVem relação a outras partes do sistema de condução $\mathrm{AV}^{3} 24283638$, esses achados sugerem que não há correlação significante entre essa alteração e o aparecimento de bloqueios AV.

Lesões no sistema His-Purkinje mostraram algumas peculiaridades. Apresença de indivíduos com HV aumentado foi significantemente maior no grupo $\mathrm{NCH}$, e pode ter ocorrido em conseqüência, provavelmente, da maior faixa etária desse grupo e da doença causadora das lesões. Estas alterações - creditadas como de pior prognóstico em estudos conduzidos em não chagásicos ${ }^{36}$ também apresentaram evolução para formas avançadas de bloqueio AV, embora com significância estatística apenas no grupo $\mathrm{CH}$. Isto leva a sugerir que chagásicos crônicos com intervalo HV aumentado teriam maior chance de apresentar bloqueios AV de graus avançados em relação aos que apresentam intervalo HV normal. Este aspecto ainda não tinha sido previamente relatado, sendo, por isso, uma contribuição que merece estudos mais aprofundados para melhor esclarecimento. Quanto à mortalidade, não houve associação com intervalo HV prolongado. Isso já é conhecido, pois, um trabalho sobre não chagásicos também não observou essa relação, embora os indivíduos com intervalo HV prolongado tivessem recebido implante de marcapasso ${ }^{6}$, sendo talvez, razão de melhor prognóstico. Contudo, o evento morte súbita no grupo $\mathrm{CH}$ ocorreu em 4 pacientes que já tinham marcapasso implantado, afastando a possibilidade de que esse tipo de tratamento possa prevenir morte súbita em todos os pacientes chagásicos crônicos.

Lesões simultâneas no nódulo $A V$ e no sistema His-Purkinje, o que representaria comprometimento mais extenso no sistema de condução $\mathrm{AV}$, estiveram presentes em pequeno número de indivíduos, prejudicando uma melhor 
análise. Contudo, mostrou-se importante em relação ao aparecimento de bloqueio AV entre os indivíduos chagásicos, pois, dos 5 com lesões em série no sistema de condução AV, 3 evoluíram para bloqueio AV. Já entre os não chagásicos, os dois nessa situação evoluíram para bloqueio $\mathrm{AV}$, sugerindo que essa associação de alterações pode evoluir para graus mais avançados de bloqueio AV. Nos dois grupos, comprometimentos mais extensos dessa região do sistema éxcitocondutor cardíaco não apresentou maior taxa de mortalidade. Tal fato ainda não foi relatado, já que os trabalhos disponíveis apresentam dados referentes a um segmento, $\mathrm{AH}$ ou HV, isoladamente ${ }^{324} 36$.

Os resultados deste estudo sugerem que a progressão para formas mais avançadas de bloqueio AV ocorreu de modo semelhante, tanto numa doença como em outra. Evidencia-se, também, que não houve diferença significante entre aqueles que tinham evidências de alterações eletrofisiológicas da condução AV previamente e que evoluíram para bloqueio AV, exceto os que tinham intervalo HV aumentado. Se se considerar a média de idade dos chagásicos, pode-se concluir que a doença de Chagas é mais grave que a esclerose primária idiopática, principalmente se, aos bloqueios $\mathrm{AV}$, forem adicionados os casos que evoluíram com disfunção do nódulo sinusal e insuficiência cardíaca, aumentando a morbidade da cardiopatia chagásica.

Nenhum achado eletrofisiológico contribuiu para identificar, de forma decisiva, aqueles com maior chance de apresentar progressão das lesões do sistema de condução AV. Contudo, a disfunção do nódulo sinusal nos chagásicos, quando eletrofisiologicamente manifesta, sugere que o seu portador venha a ter maior chance de se tornar sintomático e, a longo prazo, de necessitar de tratamento com implante de marcapasso. O intervalo HV prolongado preconiza uma evolução para formas mais avançadas de bloqueio AV em ambos os grupos. Quanto à mortalidade, não se encontraram indicadores preditivos de evolução para óbito.

Limitações no estudo. Embora se trate de um estudo prospectivo, há limitações inerentes ao longo prazo de seguimento. Alguns procedimentos hoje considerados como rotina para avaliação cardiológica não eram disponíveis no início deste projeto. Assim, protocolos agressivos de estimulação ventricular são rotineiramente utilizados atualmente para avaliar riscos de arritmia ventricular sustentada. $\mathrm{Na}$ época do início deste estudo, a indução de taquiarritmias ventriculares malignas em indivíduos assintomáticos apresentava riscos e conseqüentemente, problemas éticos; isto limitou o estudo. O número pequeno de indivíduos não chagásicos deveuse ao fato de que não é freqüente, na população de pacientes hospitalizados, a existência de portadores de bloqueio de ramo assintomáticos que estejam dentro dos critérios de inclusão. Como um grande número de mortes em indivíduos chagásicos ocorre de forma súbita, e o papel das arritmias ventriculares não foi estudado, considerações sobre este particular não puderam ser feitas.

Conclusões. 1. Os indivíduos chagásicos assintomáticos com distúrbios da condução intraventricular apresentaram maior mortalidade cardíaca súbita ou não súbita, que os indivíduos não chagásicos. 2. Os parâmetros eletrofisiológicos estudados não tiveram valor prognóstico quanto à mortalidade. 3. A presença de tempo de recuperação do nódulo sinusal aumentado foi importante indicador de futura disfunção sintomática do nódulo sinusal. 4. O intervalo HV aumentado sugeriu evolução para formas avançadas de bloqueio AV e necessidade de implante de marcapasso apenas nos indivíduos chagásicos.

\section{REFERÊNCIAS BIBLIOGRÁFICAS}

1. Altschuller H, Fischer JD, Furman S. Significance of isolated $\mathrm{H}-\mathrm{V}$ interval prolongation in symptomatic patients without documented heart block. American Heart Journal 97:19-26, 1979

2. Barrett PA, Peter CT, Swan HJC, Singh BN, Mandel WJ. The frequency and prognostic significance of electrocardiographic abnormalities in clinically norma individuals. Progress in Cardiovascular Disease 23:299$319,1981$.
3. Benchimol C B, Ginefra P, Benchimol AB. Avaliação eletrofisiológica. In: Cançado JR, Chuster M (eds) Cardiopatia chagásica. Belo Horizonte: Fundação Carlos Chagas de Pesquisas Médicas, p.213-222, 1985.

4. Cohen HC, Singer DH. Bundle branch block and other forms of aberrant intraventricular conduction: clinical aspects. In: Mandel WJ (ed) Cardiac arrhythmias. Their mechanisms, diagnosis, and management. $2^{\text {nd }}$ edition. Philadelphia, Lippincott, 413, 1987. 
5. Curti HJV, Sanches PCR, Jabur Fo M, Mazzoni CJ, Pasian S, Carvalhal SS. Morte súbita em uma população de baixo nível sócio-econômico da cidade de Campinas. Estudo anatomopatológico. Arquivos Brasileiros de Cardiologia 41:109-114, 1983.

6. Denes P, Dhingra RC, Wu D, Chuquimia R, Amat-y-León $\mathrm{F}$, Wyndham C, Rosen KM. H-V interval in patients with bifascicular block (right bundle branch block and left anterior hemiblock). Clinical, electrocardiographic, and electrophysiologic correlations. American Journal of Cardiology 35:23-29, 1975.

7. Denes P, Dhingra RC, Wu D, Wyndham CR, Amat-y-Leon $\mathrm{F}$, Rosen KM. Sudden death in patients with chronic bifascicular block. Archives of Internnal Medicine 137:1005-1021, 1977.

8. Dhingra RC, Wyndham C, Amat-y-León F, Wu D, Towne WD, Rosen KM. Significance of A-H interval in patients with chronic bundle branch block. Clinical, electrophysiologic and follow-up observations.American Journal of Cardiology 37:231-236, 1976.

9. Dhingra RC, Wyndham C, Bauerfeind R, Denes P, Wu D, Swiryn S, Rosen K. Significance of chronic bifascicular block without apparent organic heart disease. Circulation 60:33-42, 1979.

10. Dhingra RC, Wyndham $C$, Bauerfeind $R$, Swirin $S$, Deedwania PC, Smith T, Denes P, Rosen KM. Significance of block distal to the His bundle induced by atrial pacing in patients with chronic bifascicular block. Circulation 60:1455-1464, 1979.

11. Dias E, Laranja FS, Nóbrega G. Doença de Chagas. Mem Inst Oswaldo Cruz 43:520-550, 1945.

12. Dias JCP. The indeterminate form of human chronic Chagas' disease. A clinical epidemiological review. Revista da Sociedade Brasileira de Medicina Tropical 22:147-156, 1989.

13. Dias JCP. História natural da doença de Chagas. Arquivos Brasileiros de Cardiologia 65:305-311, 1995.

14. Fahy GJ, Pinski SL, Miller DP, Mccabe N, Pye C, Walsh MJ, Robinson K. Natural history of isolated bundle branch block. American Journal of Cardiology 77:1185-1190, 1996.

15. Féhér J, Pileggi F, Teixeira $V$, Tranchesi J, Pinto Lima FX, Spiritus O, Chansky M, Décourt LV. The vectorcardiogram in chronic Chagas myocarditis. An analysis of the intraventricular conduction delays associated with superiorly oriented ÂQRS. American Journal of Cardiology 5:349-357, 1960.

16. Kulbertus HE. The magnitude of risk of developing complete heart block in patients with LAD-RBBB. American Heart Journal 86:278-280, 1973.

17. Laranja FS, Dias E, Nóbrega G, Miranda A. Chagas' disease. A clinical, epidemiologic, and pathologic study. Circulation 14:1035-1060, 1956.
18. Lasser RP, Haft JI, Friedberg CK. Relationship of right bundle-branch block and marked left axis deviation (with left parietal or peri-infarction block) to complete heart block and syncope. Circulation 37:429-437, 1968.

19. Lenègre J. Etiology and pathology of bilateral bundle branch block in relation to complete heart block. Progress in Cardiovascular Disease 6:409-444, 1964.

20. Lev M. The pathology of complete atrioventricular block. Progress in Cardiovascular Disease 6:317-326, 1964.

21. Lopes ER, Capadeiro E, Borges MCC, Cançado MAP, Rocha A. Morte súbita e doença de Chagas. Análise dos fatores predisponentes do óbito súbito do chagásico crônico. Memórias do Instituto Oswaldo Cruz 77:255-262, 1982.

22. Mady C, Nacruth R. Historia natural da cardiopatia chagásica crônica: fatores prognósticos. Revista da Sociedade de Cardiologia do Estado de São Paulo 4:124132, 1994.

23. Maguire JH, Hoff R, Sherlock I, Guimarães AC, Sleigh AC, Raamos NB, Mott KE, Weller TH. Cardiac morbidity and mortality due to Chagas' disease: prospective electrocardiographic study of a Brazilian community. Circulation 75:1140-1145, 1987.

24. Maia IG, Sá RS, Loyolla LH, Cruz Fo F, Studart PCC, Amino JGC, Peixoto ECS, Drumond Neto C, Dohmann HFJ. O nódulo atrioventricular na cardiopatia chagásica crônica. Arquivos Brasileiros de Cardiologia 42:191-195, 1984.

25. McAnulty JH, Rahimtoola SH, Murphy E, Demots H, Ritzmann L, Kanarek PE, Kauffman S. Natural history of "high-risk" bundle-branch block. Final report of a prospective study. New England Journal of Medicine 307:137-143, 1982. Medicine 307:137-143, 1982.

26. Oliveira JSM, Araújo RRC, Navarro MA, Muccillo G Cardiac thrombosis and thromboembolism in chronic Chagas' heart disease. American Journal of Cardiology 52:147-151, 1983.

27. Pimenta J, Miranda M, Silva LA. Abnormal atrioventricular nodal response patterns in patients with long-term Chagas' disease. Chest 78:310-315, 1980.

28. Pimenta J, Miranda M, Pereira CB. Electrophysiologic findings in long-term asymptomatic chagasic individuals. American Heart Journal 106:374-380, 1983.

29. Porto CC. O eletrocardiograma no prognóstico e evolução da doença de Chagas. Arquivos Brasileiros de Cardiologia 17:313-346, 1965.

30. Prata A, Lopes ER, Capadeiro E. Morte súbita. In: Cançado JR, Chuster M (eds) Cardiopatia chagásica. Fundação Carlos Chagas de Pesquisa Médica, Belo Horizonte, p 114-120, 1985.

31. Ramos J, Mascarenhas A, Sporques FA, Melo HK, Borges $\mathrm{S}$, Pucci $\mathrm{H}$, lunes $M$. Eletrocardiograma na miocardite chagásica (72 casos). Arquivos Brasileiros de Cardiologia 2:145-150, 1949. 
32. Rardon DP, Milees WM, Mitrani RD, Klein LS, Zipes DP. Atrioventricular Block and Dissociation. In: Zipes DP, Jalife J (eds) Cardiac electrophysiology: from cell to bedside. $2^{\text {nd }}$ edition. Saunders, Philadelphia, p.938, 1994.

33. Rosenbaum MB, Alvarez AJ. The electrocardiogram in chronic chagasic myocarditis. American Heart Journal 50:492-527, 1955.

34. Rosenbaum MB. Chagasic myocardiopathy. Progress in Cardiovascular Diseases 7:199-225, 1964.

35. Rotman M, Triebwasser JH. Aclinical and follow-up study of right and left bundle branch block. Circulation 51:477484, 1975.

36. Scheinman MM, Peters RW, Modin G, Brennan M, Mies C, O'Young J. Prognostic value of infranodal conduction time in patients with chronic bundle branch block. Circulation 56:240-244, 1977.

37. Scherlag BJ, Lau SH, Helfant RH, Berkowitz WD, Stein E, Damato NA. Catheter technique for recording His bundle activity in man. Circulation 39:13-18, 1969.

38. Sosa EA. Contribuição para o estudo das propriedades eletrofisiológicas do coração na doença de Chagas. São Paulo. Tese de Doutorado. Faculdade de Medicina da Universidade de São Paulo, São Paulo, 1979.

39. Vicchi FL, Romero LC, Arávalo JRG. Prevalência de bloqueios de ramos e fasciculares esquerdos do feixe de His na cardiopatia chagásica. Arquivos Brasileiros de Cardiologia 39:87-88, 1982. 